\title{
Detecting copy-number variations in whole-exome sequencing data using the eXome Hidden Markov Model: an 'exome-first' approach
}

\author{
Satoko Miyatake ${ }^{1}$, Eriko Koshimizu ${ }^{1}$, Atsushi Fujita ${ }^{1}$, Ryoko Fukai ${ }^{1}$, Eri Imagawa ${ }^{1}$, Chihiro Ohba ${ }^{1}$, \\ Ichiro Kuki ${ }^{2}$, Megumi Nukui ${ }^{2}$, Atsushi Araki ${ }^{3}$, Yoshio Makita ${ }^{4}$, Tsutomu Ogata ${ }^{5}$, Mitsuko Nakashima ${ }^{1}$, \\ Yoshinori Tsurusaki ${ }^{1}$, Noriko Miyake ${ }^{1}$, Hirotomo Saitsu ${ }^{1}$ and Naomichi Matsumoto ${ }^{1}$
}

Whole-exome sequencing (WES) is becoming a standard tool for detecting nucleotide changes, and determining whether WES data can be used for the detection of copy-number variations (CNVs) is of interest. To date, several algorithms have been developed for such analyses, although verification is needed to establish if they fit well for the appropriate purpose, depending on the characteristics of each algorithm. Here, we performed WES CNV analysis using the eXome Hidden Markov Model (XHMM). We validated its performance using 27 rare CNVs previously identified by microarray as positive controls, finding that the detection rate was $59 \%$, or higher (89\%) with three or more targets. XHMM can be effectively used, especially for the detection of $>200 \mathrm{~kb}$ CNVs. XHMM may be useful for deletion breakpoint detection. Next, we applied XHMM to genetically unsolved patients, demonstrating successful identification of pathogenic CNVs: 1.5-1.9-Mb deletions involving NSD1 in patients with unknown overgrowth syndrome leading to the diagnosis of Sotos syndrome, and 6.4-Mb duplication involving MECP2 in affected brothers with late-onset spasm and progressive cerebral/cerebellar atrophy confirming the clinical suspect of $M E C P 2$ duplication syndrome. The possibility of an 'exome-first' approach for clinical genetic investigation may be considered to save the cost of multiple investigations.

Journal of Human Genetics (2015) 60, 175-182; doi:10.1038/jhg.2014.124; published online 22 January 2015

\section{INTRODUCTION}

Whole-exome sequencing (WES) is becoming a standard tool not only for genetic research but also clinical diagnosis. Compared with whole genome sequencing (WGS), it is possible to examine more than 20000 genes in the human genome in a time- and cost-effective manner with whole-exon capture methods. For this reason, WES is more often chosen than WGS, particularly in clinical settings. Although the main purpose of WES is to identify single-nucleotide variants or short indels $(<10 \mathrm{bp}$ ), recent bioinformatic advances have enabled WES data to be used more widely, for example in copy-number analysis, genotyping, or linkage studies, making it a more comprehensive genetic tool.

Copy-number variations (CNVs) are one determinant of genetic diversity, contributing to many human diseases including neuropsychiatric disorders, ${ }^{1-5}$ obesity ${ }^{6}$ and cancer. ${ }^{7}$ Microarrays have been used to detect CNVs with great accuracy, although small regions $(<10 \mathrm{~kb}$ in size) are relatively difficult to detect because of probe resolution. Thus, it would be advantageous to be able to screen CNVs using WES data before or even without performing microarray analysis, which requires time and cost. Furthermore, because WES data includes genetic information from each exonic region, there is the possibility of detecting much smaller exonic CNVs than by microarray.

For copy-number analysis, determining sequence read depth is the only method adopted for WES to date. ${ }^{8}$ However, as suspected, it is more difficult to identify CNVs with similar sensitivity and specificity using WES data than WGS data, because of the enrichment of non-contiguous regions by WES. Although several CNV calling methods have been introduced, recent studies show that none worked satisfactorily compared with WGS-based CNV calling tools. ${ }^{9,10}$ Even calls generated by WES-based CNV detection tools differ and the majority do not overlap, ${ }^{9,10}$ as they use different statistical models and analytical algorithms, and have distinct output characteristics. Sample preparation for WES or processing WES data (for example, exon capture or mapping) can also influence copy-number analyses. Furthermore, specific computational environments are required to use these tools. Considering all these factors, the tool most suitable for the purpose and WES data generated must be chosen.

The eXome Hidden Markov Model (XHMM) ${ }^{11}$ is a well-known WES-based CNV calling tool that focuses on detecting rare $(<5 \%)$

${ }^{1}$ Department of Human Genetics, Yokohama City University Graduate School of Medicine, Yokohama, Japan; ${ }^{2}$ Department of Pediatric Neurology, Pediatric Medical Care Center, Osaka City General Hospital, Osaka, Japan; ${ }^{3}$ Department of Pediatrics, Kansai Medical University, Hirakata, Japan; ${ }^{4}$ Education Center, Asahikawa Medical University, Asahikawa, Japan and ${ }^{5}$ Department of Pediatrics, Hamamatsu University School of Medicine, Hamamatsu, Japan

Correspondence: Professor N Matsumoto, Department of Human Genetics, Yokohama City University Graduate School of Medicine, 3-9 Fukuura, Kanazawa-ku, Yokohama 236-0004, Japan. 
CNVs. It uses principal component analysis to normalize read depth, filtering out extremely variable targets not derived from copy-number changes. A hidden Markov model identifies exon-resolution CNVs. Moreover, XHMM generates breakpoint quality scores, which is useful for further validation.

Here, we use XHMM for WES-based CNV detection and validate the results. We also consider the possibility of an 'exome-first' approach for detecting $\mathrm{CNVs}$ at the genome-wide level before conventional microarray method.

\section{MATERIALS AND METHODS \\ Samples}

For the XHMM validation study, exome data from 21 samples were included WES data from 100 in-house healthy Japanese individuals were used as normal controls at validation study. For applying XHMM to pedigrees having patients with undetermined genetic causes, exome data from 11 samples including patients and their parents were tested. WES data from 333 in-house healthy Japanese individuals were used as normal controls at applying stage. Two different normal control sets were prepared to match the experimental conditions (particularly for the version of the whole-exome capture kit used) between tested samples and normal controls. Experimental protocols were approved by the Committee for Ethical Issues at Yokohama City University School of Medicine. Written informed consent was obtained from all individuals or their parents.

\section{Whole-exome sequencing}

Genomic DNA was captured using the SureSelect Human All Exon kit (Agilent Technologies, Santa Clara, CA, USA). For XHMM validation, one sample was captured with v3, 106 samples (including 90 controls) with v4, 10 control samples with v4+UTRs and four samples with v5. For XHMM application, 344 samples (including 333 controls) were captured with v5. Samples were sequenced on either the Illumina GAIIx platform (Illumina, San Diego, CA, USA) with 108-bp paired-end reads or HiSeq2000 or HiSeq2500 (Illumina, San Diego, CA, USA) with 101-bp paired-end reads. Image analysis and base calling were performed using sequence control software with real-time analysis and CASAVA software (Illumina). Reads were aligned to GRCh37 using Novoalign (http://www.novocraft.com/). Marking PCR duplicates, indel realignment and base-quality-score recalibration were performed using Picard (http://picard. sourceforge.net/) and Genome Analysis ToolKit (GATK) (https://www.broadinstitute.org/gatk/index.php). For 21 samples and 100 in-house normal controls, the WES mean coverage depth against RefSeq coding sequence was $120.5 \times$ and $92.1 \times$, respectively, and 92.3 and $93.2 \%$ of total coding sequence bases covered by 15 reads or more, respectively.

\section{CNV analysis}

Copy-number analysis was performed using the default settings of eXome Hidden Markov Model v1.0 (XHMM), a statistical tool for copy-number analysis. ${ }^{11}$ In brief, we first calculated coverage depth using analysis-ready BAM files generated from the Novoalign/Picard/GATK data-processing pipeline, following GATK best practices (https://www.broadinstitute.org/gatk/guide/ best-practices). GATK depth of coverage was performed using the same parameters as previously described. ${ }^{12}$ After calculation, the mean coverage for each exon target of each sample was merged into a single samples-by-target matrix. ${ }^{11}$ To call CNVs, the following downstream analyses were performed using previously described commands. ${ }^{12}$ For the validation study, the 21 tested exomes were analyzed with 100 in-house normal control exomes. For applying XHMM to undiagnosed pedigrees, patients and parents were compared with 333 in-house normal control exomes. Principal component analysis-normalized and filtered $z$-scores for the whole read depth were obtained, and SignalMap Version 1.9.0.05 (Roche Nimblegen, Madison, WI, USA) used for visualization purposes.

\section{Microarray analysis}

Either Affymetrix CytoScan HD or Human Mapping $250 \mathrm{~K}$ Nsp Array for Cytogenetics (Affymetrix, Santa Clara, CA, USA) were used for all the
21 samples. To detect rare CNVs, the following criteria was used: (1) deletion must have $\geqslant 20$ marker counts and be $\geqslant 10 \mathrm{~kb}$ in size; (2) duplication must have $\geqslant 20$ marker counts and be $\geqslant 100 \mathrm{~kb}$ in size; and (3) CNV is not within a common CNV region registered in the Database of Genomic Variants (http:// dgv.tcag.ca/dgv/app/home). CNV call was confirmed by quantitative real-time PCR ( $\mathrm{PPCR})$, breakpoint PCR analysis or manual inspection of array data with high confidence values $(>90 \%)$. Through this workflow, we previously identified 27 rare CNVs in the 21 samples, which were used for positive controls for the XHMM validation in this study (Supplementary Table 1).

\section{Quantitative real-time PCR analysis}

To confirm copy-number change in suspected CNVs, qPCR of the region was performed using genomic DNA and the QuantiFast SYBR Green PCR kit (Qiagen, Hilden, Germany) on a Rotor-Gene Q (Qiagen). Relative ratios of genomic DNA copy number were calculated by comparing autosomal internal control loci (FBN1 and STXBP1) between tested individual and normal control samples, using Rotor-Gene Q software (Qiagen) and the standard curve method. Duplicate experiments were performed.

\section{Breakpoint PCR analysis}

Breakpoint PCR was performed using primers designed adjacent to detected CNVs at both $5^{\prime}$ and $3^{\prime}$ sides. PCR primers and settings are available upon request. PCR amplicons were sequenced by the Sanger method.

\section{RESULTS}

\section{Detection of known rare CNVs using XHMM}

The mean CNV size was $404.6 \mathrm{~kb}$, and 16 loci (59\%) were called by XHMM (Figure 1) (Table 1). One CNV not automatically called by XHMM was detected by manual inspection, thus the detection rate increased to $63 \%$ (17/27). The detection rate varied depending on the size of the target $\mathrm{CNV}$. For CNVs $<100 \mathrm{~kb}$, only $2 / 11$ were called (18\%). For CNVs of $100-200 \mathrm{~kb}$ or $>200 \mathrm{~kb}$, the detection rate was $75 \%(3 / 4)$ or $92 \%(11 / 12)$, respectively. Regarding CNVs uncalled by XHMM, it was frequently observed that these loci had read-depth information of two or less exon targets. There were 18 loci with three or more targets within CNVs, and among them, 16 loci (89\%) were called by XHMM. Detection rates were higher for $\mathrm{CNVs}<100 \mathrm{~kb}$ $(67 \%)$ or $100-200 \mathrm{~kb}(100 \%)$, but similar for those $>200 \mathrm{~kb}(92 \%)$.

\section{Potential CNVs called by XHMM}

A total of $184 \mathrm{CNVs}$ were automatically called by XHMM $(\sim 8.8 \mathrm{CNVs}$ per sample), including 108 deletions and 76 duplications. Of these, $41(22.2 \%)$ were $<10 \mathrm{~kb}, 91(49.5 \%)$ were $10-100 \mathrm{~kb}$ and $52(28.3 \%)$ were $>100 \mathrm{~kb}$. Among CNVs of $10-100 \mathrm{~kb}$ in size, those of $20-30 \mathrm{~kb}$ were most detected in deletions, and those of $10-20 \mathrm{~kb}$ in duplications, showing that XHMM tends to detect smaller CNVs than microarrays (Figure 2).

\section{True-positive rate of CNVs called by XHMM}

To validate the true-positive rate of calls generated by XHMM, we identified rare $\mathrm{CNV}$ s with high-quality scores and performed qPCR. Because more than half of the calls were $<100 \mathrm{~kb}$ in size, they may not be detected by microarray. Furthermore, it is unrealistic to validate all 184 loci by qPCR. Therefore, we chose CNVs on the basis of the following criteria: (1) quality score $(Q) \geqslant 65$; (2) exclude CNVs on sex chromosomes; (3) CNVs outside segmental duplications; and (4) CNVs imperfectly $(<100 \%)$ overlap with common CNVs registered in the Database of Genomic Variants. There were $14 \mathrm{CNVs}$ from eight samples satisfying the above criteria, and 6/14 were successfully confirmed by qPCR (42.9\%). In 5/8 samples, the truepositive rate was $100 \%$ (one to two CNVs in each of the five samples), although three samples showed true-positive rates of $0 \%$ (that is, were 


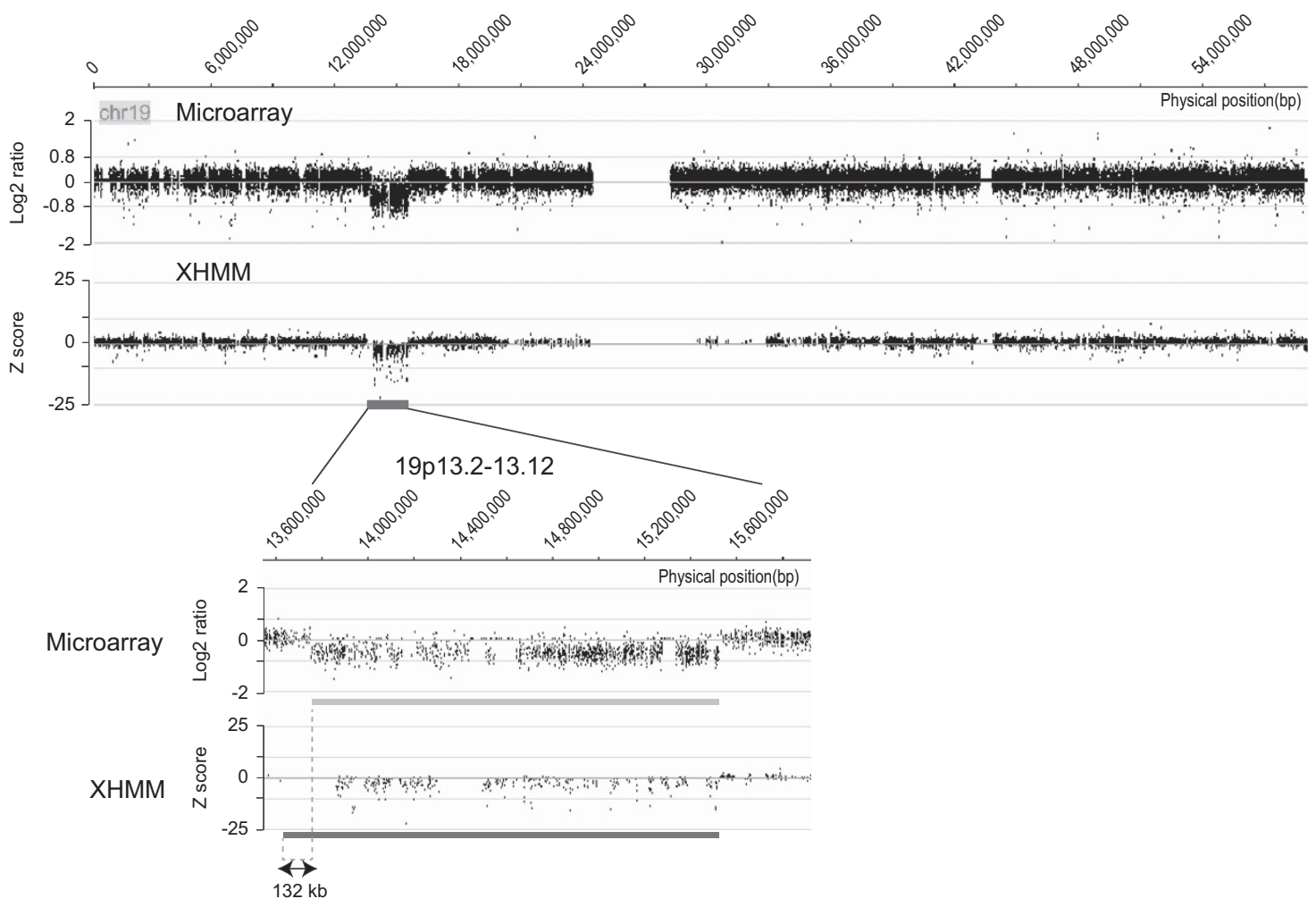

Figure 1 XHMM analysis of a 1.7-Mb deletion. Upper panel shows $\log _{2}$ ratio data obtained from microarray analysis of a sample with a 1.7-Mb deletion at 19q13.2-13.12 (chr19:13749400-15521767) identified. $X$ axis shows the physical position and y axis $\log _{2}$ ratios. Middle panel shows entire read-depth $z$-scores obtained from XHMM. X axis shows the physical position and y axis $z$-scores. XHMM CNV analysis using WES data of the sample automatically called this deletion, which is also easily detected by manual inspection. Lower panel shows enlarged view of the deleted region (upper, microarray; lower, XHMM). Because of limited read information around the distal deletion breakpoint, the distal breakpoint was shifted $132 \mathrm{~kb}$ to the distal side by XHMM compared with microarray. CNV, copy-number variation; WES, whole-exome sequencing; XHMM, eXome Hidden Markov Model. A full color version of this figure is available at the Journal of Human Genetics journal online.

Table 1 XHMM detection rates for known CNVs

\begin{tabular}{|c|c|c|c|c|c|c|}
\hline & No. of CNVs & Mean CNV size $(k b)$ & No. of detected CNVs & Detection rate (\%) & No. of detected CNVs & Detection rate (\%) \\
\hline Total & 27 & 404.6 & 16 & 59 & 17 & 63 \\
\hline$<100 \mathrm{~kb}$ & 11 & 52.7 & 2 & 18 & 2 & 18 \\
\hline $100-200 \mathrm{~kb}$ & 4 & 126.5 & 3 & 75 & 3 & 75 \\
\hline \multicolumn{7}{|c|}{ CNVs with 3 or more targets } \\
\hline Total & 18 & 581.3 & 16 & 89 & 17 & 94 \\
\hline$<100 \mathrm{~kb}$ & 3 & 76.6 & 2 & 67 & 2 & 67 \\
\hline $100-200 \mathrm{~kb}$ & 3 & 132.2 & 3 & 100 & 3 & 100 \\
\hline$>200 \mathrm{~kb}$ & 12 & 819.8 & 11 & 92 & 12 & 100 \\
\hline \multicolumn{7}{|l|}{ Gain } \\
\hline Total & 8 & 625.6 & 6 & 75 & 7 & 88 \\
\hline
\end{tabular}

Abbreviations: CNV, copy-number variation; XHMM, eXome Hidden Markov Model. 
Deletion
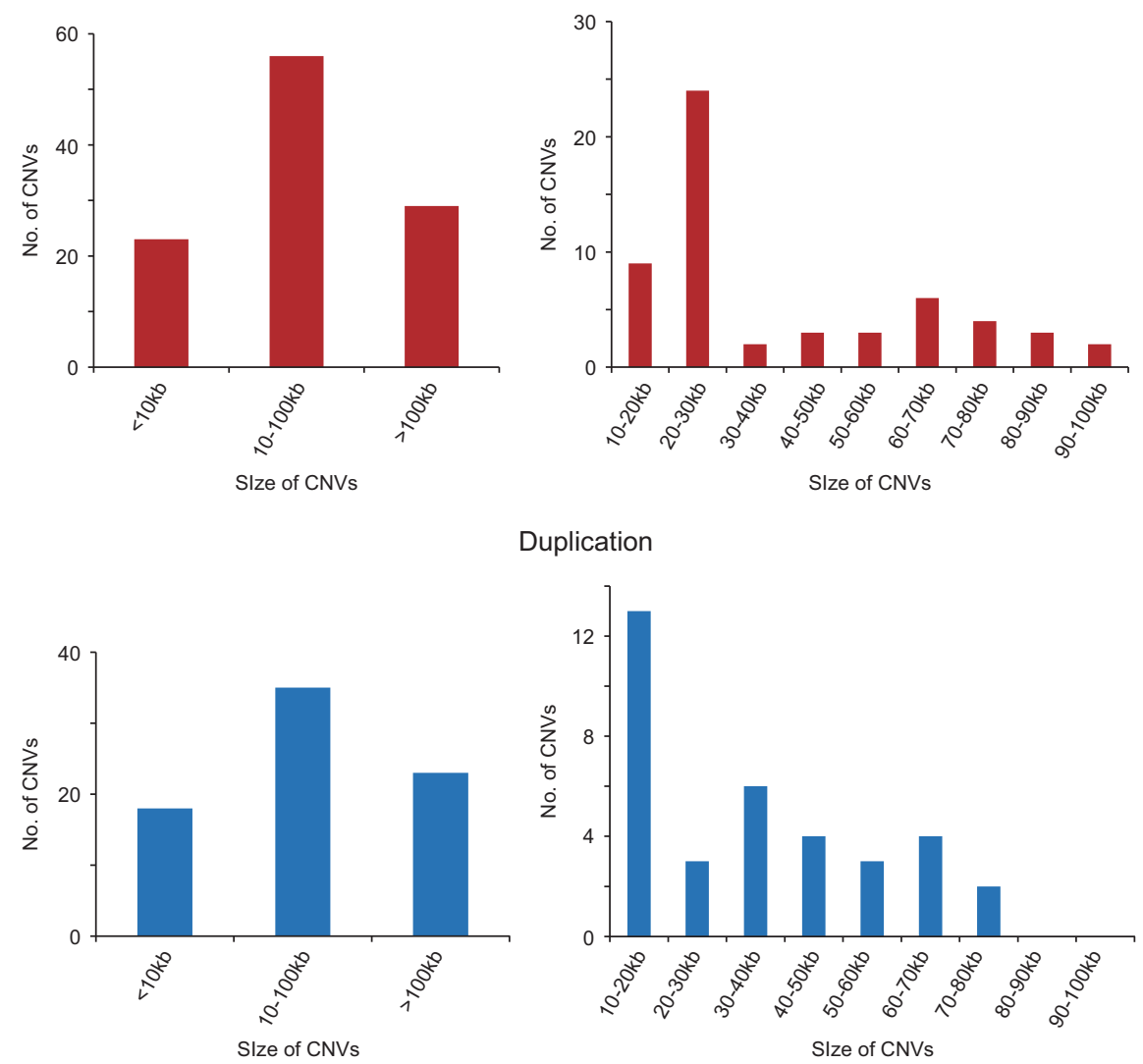

Duplication

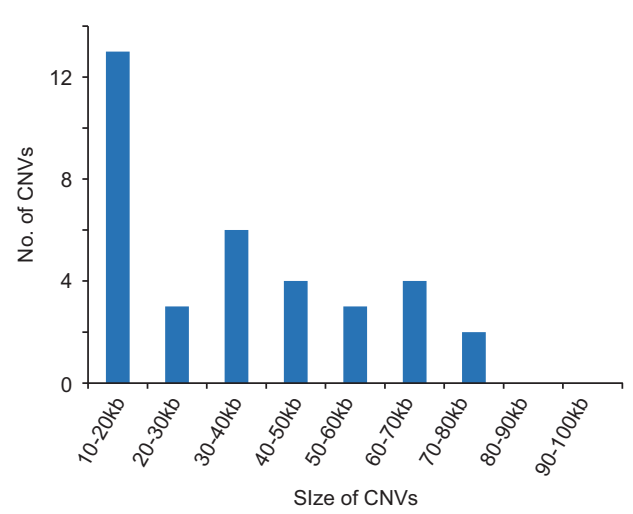

Figure 2 Overview of CNVs detected by XHMM. In all the four graphs, $x$ axis shows the size of detected CNVs and $y$ axis shows the number of CNVs. Left graphs demonstrate the size distribution of deletions (upper, red) and duplications (lower, blue). Right graphs show in detail, size distribution for 10-100 kb deletions (upper, red) and duplications (lower, blue). CNV, copy-number variation; XHMM, eXome Hidden Markov Model.

Table 2 True-positive rates for CNVs detected by XHMM

\begin{tabular}{lcccc}
\hline Sample no. & $\begin{array}{c}\text { No. of called } \\
\text { rare CNVs }\end{array}$ & $\begin{array}{c}\text { No. of confirmed } \\
\text { CNVs }\end{array}$ & $\begin{array}{c}\text { True-positive } \\
\text { rate }\end{array}$ & $\begin{array}{c}\text { Average quality } \\
\text { score of all calls }\end{array}$ \\
\hline 1 & 5 & 0 & 0 & 86.2 \\
2 & 1 & 1 & 1 & 99 \\
3 & 1 & 1 & 1 & 70 \\
4 & 2 & 0 & 0 & 85.5 \\
5 & 1 & 0 & 0 & 99 \\
6 & 1 & 1 & 1 & 89 \\
7 & 2 & 2 & 1 & 87.5 \\
8 & 1 & 1 & 1 & 99 \\
Total & 14 & 6 & 0.429 & 89.4 \\
\hline
\end{tabular}

Abbreviations: CNV, copy-number variation; XHMM, eXome Hidden Markov Model.

false positives) $(0 / 5 \mathrm{CNVs}, 0 / 2 \mathrm{CNVs}$ and $0 / 1 \mathrm{CNV}$ in each of the three samples). Average quality scores in the three negative samples were not different from that of the five positive samples (Table 2). To determine whether the true-positive call is affected by different experimental conditions, such as use of different exon capture kits or DNA from different tissue types (lymphoblastoid cell line vs blood leukocyte), we recaptured the three samples (which were previously analyzed using different capture kits) using the SureSelect Human All Exon v5 kit and genomic DNA obtained from blood, and sequenced them all in the same lane. We then performed XHMM using WES data from 333 in-house normal controls, also captured using the SureSelect Human All Exon v5 kit. All eight loci from the three samples remained uncalled by XHMM.

\section{Identification of deletion breakpoints using XHMM}

It is possible to identify breakpoints using read information surrounding deletions. Breakpoints of two deletions were successfully determined at the nucleotide level using XHMM CNV data as follows. Deletions were confirmed by qPCR, and then PCR primers designed outside the assumed deletion ends. First, a 7-kb deletion at $14 \mathrm{q} 22.3$ was determined. XHMM detected a $6.87-\mathrm{kb}$ deletion (chr14:57075873-57082744). Breakpoint PCR revealed a 7066-bp exonic deletion inTMEM260 (chr14:57075504-57082570), with one nucleotide inserted at the breakpoint, suggesting involvement of nonhomologous end joining (Figures $3 \mathrm{a}$ and $\mathrm{b}$ ). Second, a 22-kb deletion at $19 \mathrm{q} 13.33$ was determined. XHMM detected a 22.87-kb deletion (chr19:49183627-49206494). Breakpoint PCR and sequencing successfully identified a 22945-bp deletion (chr19:49183676-49206620). The proximal breakpoint was located within the last exon of SEC1P (noncoding gene), and distal breakpoint in the same orientation within a coding exon of FUT2, suggesting that a SEC1P-FUT2 fusion gene may be generated (Figures $3 \mathrm{c}$ and $\mathrm{d}$ ). This locus is a known deletion site registered in the Database of Genomic Variants with adjacent and $\sim 220$-bp self chains (human chained self-alignments archived in the UCSC Genome Browser), a novel group of short low-copy repeats. ${ }^{13}$ As the breakpoints are located within self-chain 
a

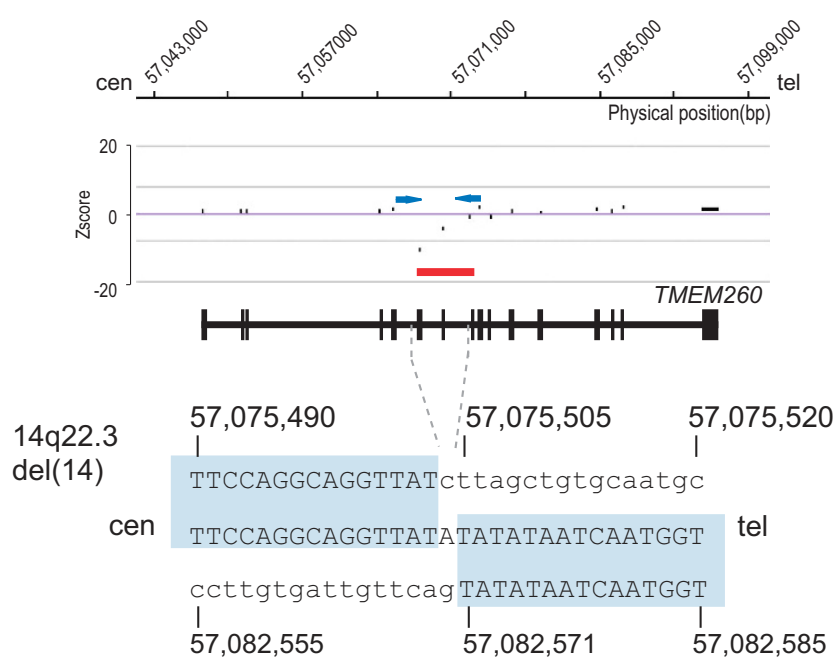

b

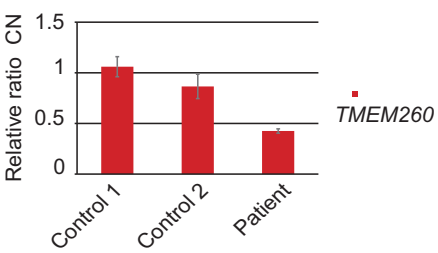

C

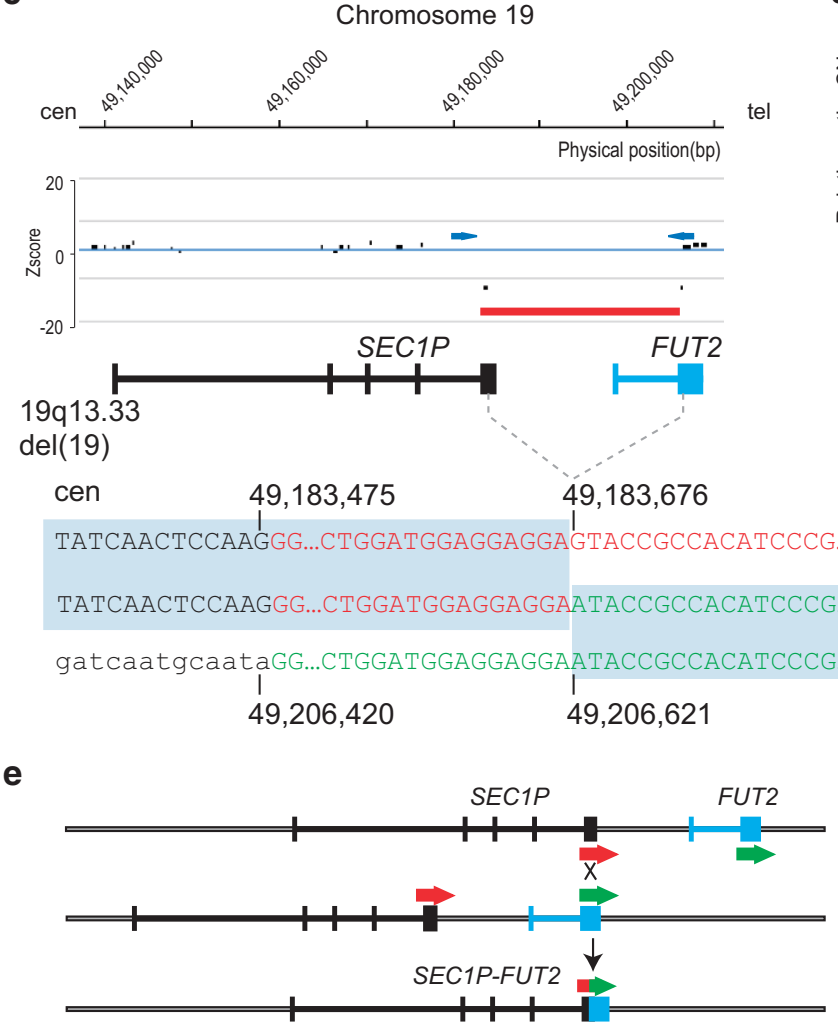

d

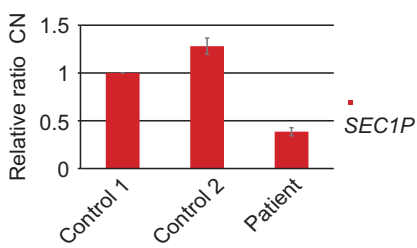

$49,183,695$ tel

Figure 3 Identification of deletion breakpoints. Deletion breakpoints were identified using XHMM. (a) Upper panel depicts a 6.87-kb deletion at 14q22.3 detected by XHMM. X axis shows the physical position and y axis $z$-score for each target. Blue arrows show forward/reverse primers designed adjacent to deletion breakpoints, and the red bar represents XHMM call for the deletion. Some TMEM2O exons are within the deletion. Lower panel represents deletion junction sequence. Upper and lower are for reference sequences covering proximal and distal deletion breakpoints, respectively. Middle shows deletion junction sequence obtained from a patient. Light blue shadow shows matched sequences. Breakpoint PCR identified exonic deletion of TMEM260. (b) Deletion at 14q22.3 detected by XHMM was confirmed by qPCR. The patient was compared with two unrelated normal controls. Relative ratio CN, relative ratio of copy number. (c) Upper panel depicts a 22.87-kb deletion at 19q13.33 detected by XHMM. X axis shows the physical position and y axis $z$-score for each target. Blue arrows show forward/reverse primers, and the red bar represents XHMM call for the deletion. The last exon of SEC1P and coding exon of FUT2 (colored in light blue) are within the region. Middle panel shows deletion junction sequences. Upper and lower are for reference sequences covering proximal and distal deletion breakpoints, respectively. Middle shows deletion junction sequence obtained from a patient. Light blue shadow shows matched sequences. Sequences in red or green represent the self chain. Breakpoint PCR identified the proximal breakpoint in the last exon of SEC1P, and distal breakpoint in a coding exon of FUT2. (d) Deletion at 19q13.33 detected by XHMM was confirmed by qPCR. The patient was compared with two unrelated normal controls. Relative ratio CN, relative ratio of copy number. (e) Schematic of suspected NAHR at this locus. Red and green arrows indicate separate self-chain repeats in SEC1P and FUT2 loci. As shown in c, the breakpoint occurs within a self-chain repeat. NAHR is likely to be involved. NAHR, non-allelic homologous recombination; XHMM, eXome Hidden Markov Model. 
a

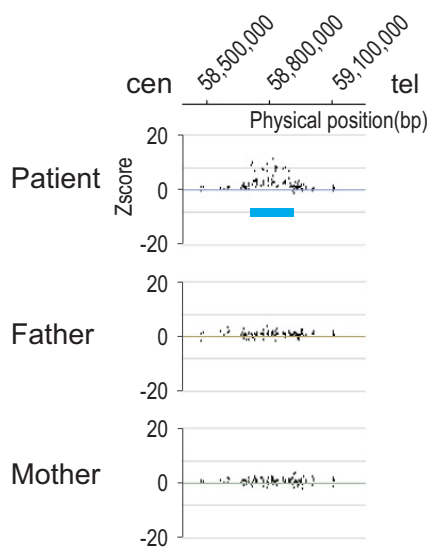

Chromosome 14

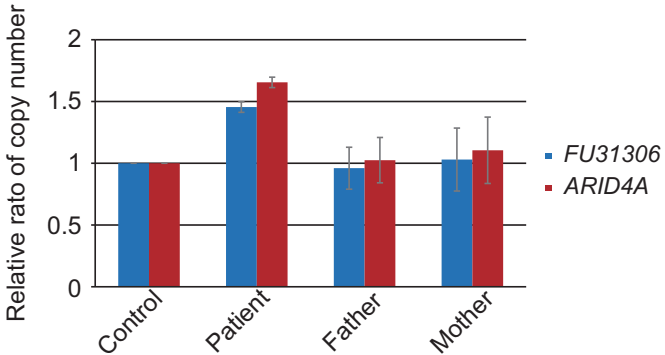

b
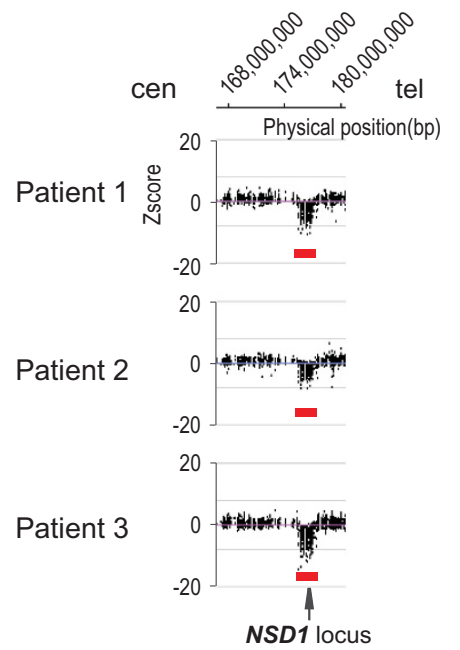

Chromosome 5

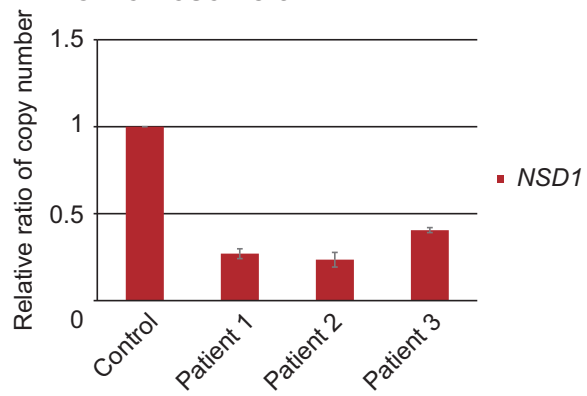

C

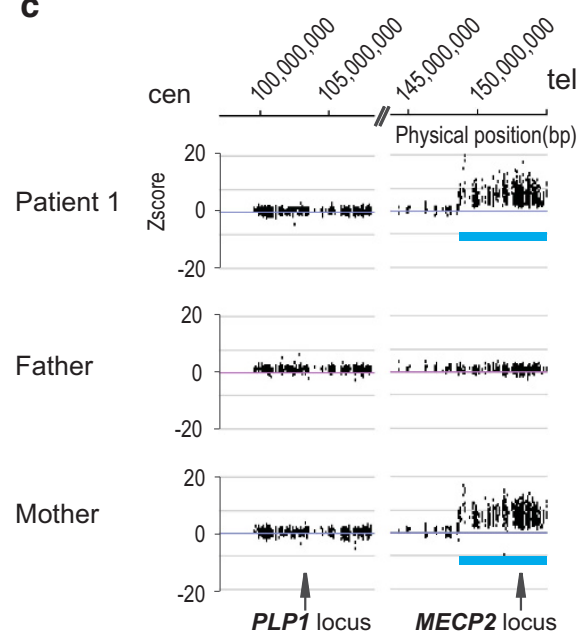

\section{Chromosome X}

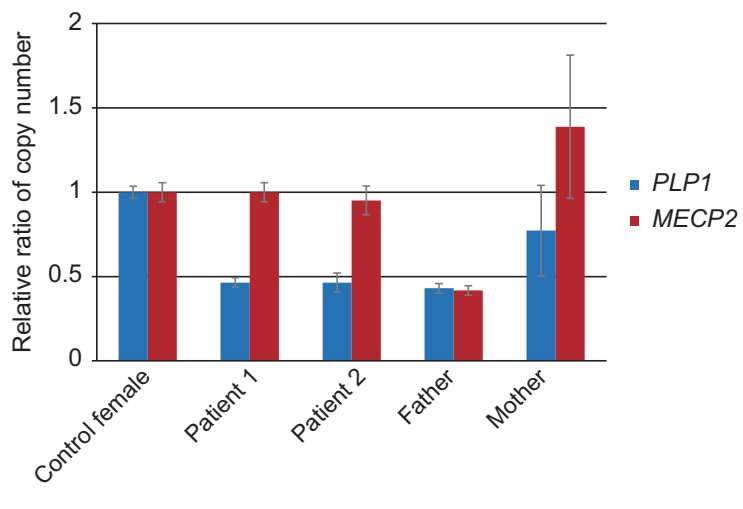

Figure 4 Examples of successful identification of pathogenic CNVs in clinical exomes. For (a-c), left panels show XHMM CNV detection. X axis shows the physical position and y axis z-score for each target. Blue and red bars represent XHMM call for duplications and deletions, respectively. Right panels show qPCR confirmation of detected CNVs. (a) Shows 184-kb duplication. Left panel shows 184-kb duplicated region at $14 \mathrm{q} 23.1$ in the patient only. De novo duplication was confirmed by qPCR of the patient, parents and an unrelated normal control. Primers for qPCR were designed separately for FU31306 and ARID4A located within the duplicated region. (b) Shows 1.5-1.9 Mb deletion at 5q35.2-35.3 involving NSD1. Left panel shows 1.5-Mb deletion in Patients 1 and 2, and 1.9-Mb deletion in Patient 3. Using qPCR, it was confirmed that the three patients show the NSD1 deletion, but not a control. (c) Shows 6.4-Mb duplication. Left panel shows 6.4-Mb duplication at Xq28 in the affected younger brother (Patient 1) and mother. By qPCR of the patient, his affected brother, their parents and an unrelated control female, it was confirmed that the patient and his affected brother (Patient 2) had two copies of MECP2 and a single copy of PLP1. Their mother had three copies of MECP2 and two copies of PLP1. The control female had two copies of both MECP2 and PLP1, and their unaffected father had one copy of both MECP2 and PLP1. Primers were separately designed for MECP2 and PLP1. PLP1 can be used as an internal control of $\mathrm{X}$ chromosome. CNV, copy-number variation; XHMM, eXome Hidden Markov Model. 
repeats, non-allelic homologous recombination is the likely mechanism to induce this deletion with acquired genomic instability (Figure 3e).

\section{Successful identification of CNVs by an 'exome-first' approach} We used XHMM for an 'exome-first' approach for genetically undiagnosed patients. Called pathological CNVs were subsequently confirmed by qPCR.

A $184-k b$ duplication in a patient with severe intellectual disability. WES analysis of a patient with severe intellectual disability did not identify any causative mutations. However, XHMM detected a 184-kb duplication at 14q23.1 (chr14:58711513-58896203). The de novo duplication was confirmed by $\mathrm{qPCR}$ using DNA from the patient and her parents (Figure 4a). A de novo deletion at 14q23.1-q23.3 was previously reported in a patient with multiple congenital anomalies and intellectual disability, ${ }^{14}$ suggesting the $14 \mathrm{q} 23.1$ duplication may be linked to intellectual disability in this patient.

The 1.5-1.9-Mb deletions involving NSD1 in patients with unknown overgrowth syndrome. WES of five patients manifesting overgrowth did not identify any associated pathological variants. Using XHMM, $3 / 5$ patients were found to have large deletions involving NSD1 at $5 \mathrm{q} 35.2-35.3$ (1.5-Mb deletion in two and 1.9-Mb deletion in one patient), which were confirmed by qPCR (Figure $4 \mathrm{~b}$ ). The patients were subsequently diagnosed with Sotos syndrome. These results clearly demonstrate the usefulness of XHMM, especially for large CNVs $(>200 \mathrm{~kb})$

A 6.4-Mb duplication involving MECP2 in affected brothers with lateonset spasm accompanied by progressive cerebral/cerebellar atrophy. WES of a 7-year-old younger brother and his parents revealed no candidate mutation. His clinical features implied MECP2 duplication, and trio-based XHMM did indeed identify a 6.4-Mb duplication harboring MECP2 in the patient and his mother. Using $\mathrm{qPCR}$, the patient and his elder affected brother were found to have two MECP2 copies, while his mother and father had three copies and one copy, respectively (Figure 4c).

\section{DISCUSSION}

We characterized detection rate on the basis of the size of known CNVs and applied XHMM to genetically unsolved patients. We also demonstrated the feasibility of detecting deletion breakpoints and diagnostic CNV.

The overall detection rate for known CNVs was as high as 59\%, and even higher $(89 \%)$ with three or more targets, compatible with an original report stating that sensitivity for $\mathrm{CNV}$ s overlapping three to five targets was $79-85 \% .{ }^{11}$ Accordingly, larger CNV size increases the detection rate, and in general, $\mathrm{CNVs}>200 \mathrm{~kb}$ are likely to be detected.

Overall, XHMM tended to call smaller CNVs than microarrays. In this study, $22.2 \%$ of total calls were $<10 \mathrm{~kb}$ and $71.7 \%<100 \mathrm{~kb}$, again similar to the original paper, ${ }^{11}$ and indicating it may be possible to detect exonic deletions. However, we found that about half of these calls were false positives, regardless of the quality score. Because falsepositive calls can be diminished by unifying exome capture methods or DNA quality used for WES, it may be important to minimize conditional experimental differences for higher true-positive rates. It is reported that XHMM performance depends on the reference standards used, ${ }^{15}$ as we also experienced in our study.

Although adjacent read information is usually limited, XHMM data can be useful for identifying $\mathrm{CNV}$ breakpoints. We were able to successfully determine breakpoints of 7 - and 22-kb deletions, which are usually difficult to detect by microarrays. CNV calls that perfectly overlap with nonpathogenic common CNVs are usually considered low priority. As shown here at the 22-kb deletion, CNV may generate a new fusion gene. Identifying breakpoints can provide precise information, including in-frame gene fusions.

We also demonstrated that pathogenic CNVs can be detected by XHMM using WES data, leading to appropriate clinical diagnosis. As it is not known whether a nucleotide variant or gross $\mathrm{CNV}$ is the culprit before analysis, an 'exome-first' approach may save time and cost of unnecessary microarray analysis. This approach is even more effective with target CNVs $>200 \mathrm{~kb}$.

In conclusion, XHMM can be effectively used, especially for detection of $>200 \mathrm{~kb}$ CNVs. Although the CNV detection rate is not optimal, this method can save unnecessary investigation.

\section{CONFLICT OF INTEREST}

The authors declare no conflict of interest.

\section{ACKNOWLEDGEMENTS}

We thank all the participants for their cooperation in this research. We also thank Dr K. Nishiyama, Ms K. Takabe, Mr T. Miyama, Ms N. Watanabe, Ms A. Sato and Ms S. Sugimoto from the Department of Human Genetics, Yokohama City University Graduate School of Medicine, and Dr D. Yamaguchi from BITS Co., Ltd., for their technical assistance. This work was supported by the Ministry of Health, Labour and Welfare of Japan (NMi, NMa); the Japan Society for the Promotion of Science (a Grant-in-Aid for Scientific Research (C) (26461549) (SM), a Grant-in-Aid for Scientific Research (B) (25293085, 25293235) (NMi, HS), a Grant-in-Aid for Scientific Research (A) (13313587) (NMa) and a Grant-in-Aid for Challenging Exploratory Research (26670505) (HS)); the Takeda Science Foundation (NMi, NMa, HS); the Fund for Creation of Innovation Centers for Advanced Interdisciplinary Research Areas Program in the Project for Developing Innovation Systems (NMa); the Strategic Research Program for Brain Sciences (11105137) (NMa); and a Grant-in-Aid for Scientific Research on Innovative Areas (Transcription Cycle) from the Ministry of Education, Culture, Sports, Science and Technology of Japan (12024421) (NMi, NMa).

1 Pinto, D., Pagnamenta, A. T., Klei, L., Anney, R., Merico, D., Regan, R. et al. Functional impact of global rare copy number variation in autism spectrum disorders. Nature 466, 368-372 (2010).

2 Stefansson, H., Rujescu, D., Cichon, S., Pietilainen, O. P., Ingason, A., Steinberg, S. et al. Large recurrent microdeletions associated with schizophrenia. Nature 455, 232-236 (2008).

3 Cook, E. H. Jr \& Scherer, S. W. Copy-number variations associated with neuropsychiatric conditions. Nature 455, 919-923 (2008).

4 Jakobsson, M., Scholz, S. W., Scheet, P., Gibbs, J. R., VanLiere, J. M., Fung, H. C. et al. Genotype, haplotype and copy-number variation in worldwide human populations. Nature 451, 998-1003 (2008)

5 Heinzen, E. L., Radtke, R. A., Urban, T. J., Cavalleri, G. L., Depondt, C., Need, A. C. et al. Rare deletions at $16 \mathrm{p} 13.11$ predispose to a diverse spectrum of sporadic epilepsy syndromes. Am. J. Hum. Genet. 86, 707-718 (2010).

6 Bochukova, E. G., Huang, N., Keogh, J., Henning, E., Purmann, C., Blaszczyk, K. et al. Large, rare chromosomal deletions associated with severe early-onset obesity. Nature 463, 666-670 (2010).

7 Beroukhim, R., Mermel, C. H., Porter, D., Wei, G., Raychaudhuri, S., Donovan, J. et al. The landscape of somatic copy-number alteration across human cancers. Nature 463, 899-905 (2010).

8 Zhao, M., Wang, Q., Jia, P. \& Zhao, Z. Computational tools for copy number variation (CNV) detection using next-generation sequencing data: features and perspectives. BMC Bioinformatics. 14(Suppl 11), S1 (2013).

9 Tan, R., Wang, Y., Kleinstein, S. E., Liu, Y., Zhu, X., Guo, H. et al. An evaluation of copy number variation detection tools from whole-exome sequencing data. Hum. Mutat. 35, 899-907 (2014).

10 Kadalayil, L., Rafiq, S., Rose-Zerilli, M. J., Pengelly, R. J., Parker, H., Oscier, D. et al. Exome sequence read depth methods for identifying copy number changes. Brief Bioinform. (e-pub ahead of print 28 August 2014). 
11 Fromer, M., Moran, J. L., Chambert, K., Banks, E., Bergen, S. E., Ruderfer, D. M. et al. Discovery and statistical genotyping of copy-number variation from whole-exome sequencing depth. Am. J. Hum. Genet. 91, 597-607 (2012).

12 Fromer, M. \& Purcell, S. M. Using XHMM software to detect copy number variation in whole-exome sequencing data. Curr. Protoc. Hum. Genet. 81, 7.23.1-7.23.21 (2014)

13 Zhou, W., Zhang, F., Chen, X., Shen, Y., Lupski, J. R. \& Jin, L. Increased genome instability in human DNA segments with self-chains: homology-induced structural variations via replicative mechanisms. Hum. Mol. Genet. 22 2642-2651 (2013).

14 Lehalle, D., Sanlaville, D., Guimier, A., Plouvier, E., Leblanc, T., Galmiche, L. et al. Multiple congenital anomalies-intellectual disability (MCA-ID) and neuroblastoma in a patient harboring a de novo 14q23.1q23.3 deletion. Am. J. Med. Genet. A 164A, 1310-1317 (2014). 15 Magi, A., Tattini, L., Cifola, I., D'Aurizio, R., Benelli, M., Mangano, E. et al. EXCAVATOR: detecting copy number variants from whole-exome sequencing data. Genome Biol. 14, R120 (2013)

Supplementary Information accompanies the paper on Journal of Human Genetics website (http://www.nature.com/jhg) 